\title{
Representación de áreas temáticas en las revistas latinoamericanas de Bibliotecología y Ciencia de la información en español (2008-2016)
}

\section{Ignacio Mancini}

Universidad de Buenos Aires, Facultad de Filosofía y Letras, Instituto de Investigaciones Bibliotecológicas (INIBI), Argentina | ignaciomancini@gmail.com / https://orcid.org/oooo-0003-3381-461X

\begin{abstract}
Manuel Riveiro
Universidad de Buenos Aires, Facultad de Ciencias Sociales, Instituto de Investigaciones Gino Germani (IIGG). Universidad Nacional Arturo Jaureche. Instituto de Ciencias de la Salud. Universidad de Buenos Aires, Facultad de Filosofía y Letras, Instituto de Investigaciones Bibliotecológicas (INIBI), Argentina | manox3@gmail.com / http://orcid.org/oooo-0003-4653-2902
\end{abstract}

\section{Ivalú Ramírez Ibarra}

Universidad de Buenos Aires, Facultad de Filosofía y Letras, Instituto de Investigaciones Bibliotecológicas (INIBI), Argentina | iramirezi@filo.uba.ar

\section{Resumen}

Introducción: Las revistas de investigación constituyen el núcleo del campo de conocimiento de una disciplina científica. En el caso de las disciplinas con gran desarrollo del ejercicio profesional, también conforman este núcleo algunas revistas menos rigurosas con respecto a la naturaleza de sus contenidos y sus requisitos formales. Objetivo: Describir perfiles temáticos por revista y país a partir del análisis de artículos de las revistas latinoamericanas de Bibliotecología en español (2008-2016). Se busca detectar tendencias locales e identificar áreas de vacancia. Metodología: A través un análisis cuantitativo básico se caracterizan las revistas latinoamericanas de Bibliotecología en español para el período señalado. Se elabora una base de datos con los artículos de las publicaciones identificadas. Resultados: Se identificaron un total de 28 títulos de revistas y 2388 artículos. Se señala disparidad entre países en la cantidad de títulos y artículos. No se identifican tendencias temáticas significativas por subregión.

Subject areas in Library and Information Science Latin American journals in Spanish language (2008-2016)

\footnotetext{
Abstract

Introduction: Research journals form the core of the field of knowledge of a scientific discipline. In the case of profession-oriented disciplines, some "not-as-scientific-as-others" journals also define this core. Objectives: To Describe thematic profiles
}

Palabras clave

Bibliotecología y Ciencia de la Información Revistas científicas

Bibliometría

Clasificación del conocimiento América Latina

Keywords

Library and Information Science Scientific Journals

Bibliometrics

Knowledge Organisation

Latin America 
by journal and country from the analysis of articles of Latin American Librarianship and Information Science (LIS) journals in Spanish language (2008-2016), and to detect local trends and identify areas of vacancy. Methodology: Through a basic quantitative analysis, Latin American LIS Journals are characterized for the period indicated. A database with the articles of the publications identified is made. Results: A total of 28 journal titles and 2388 articles have been collected. Disparity between countries is identified in the quantities of titles and articles. No significant thematic trends are identified by subregion.

Artículo recibido: 2-11-2018. Aceptado: 25-03-2019

\title{
Introducción ${ }^{1}$
}

\begin{abstract}
Los espacios para la reflexión sobre la investigación son escenarios propicios para mirarnos a nosotros mismos y para reflexionar sobre todos los aspectos vinculados a la Bibliotecología y Ciencia de la Información como disciplina y como profesión. Si nosotros no construimos y consolidamos estos espacios en los que proyectar la mirada hacia la reflexión y el debate en torno a nuestro propio campo disciplinar, nadie lo hará por nosotros... Conocer nuestra propia disciplina, debatir sobre los temas, los métodos, las prácticas de comunicación, la formación en investigación y todos los aspectos que están vinculados con el desarrollo y consolidación de la $\mathrm{ByCl}$ es un aspecto nuclear que nos preocupa y ocupa a todos los que formamos parte de la comunidad académica y profesional en este dominio de conocimiento.
\end{abstract}

Sandra Miguel, 2016, p. 8

1. Este artículo es producto del Proyecto de Reconocimiento Institucional para Graduados, Programación Científica 2016-2017 del Instituto de Investigacione Bibliotecológicas de la Facultad de Filosofía y Letras de la Universidad de Buenos Aires. Representación de áreas temáticas en las revistas latinoamericanas de Bibliotecología de lengua castellana (2008-2016). Director: Ignacio Mancini; codirector: Manuel Riveiro; integrantes: Ivalú Ramírez Ibarra, Mariano Barberán Zangaro, Mariano Corujo, Sebastián González, Verónica Saquilán. Un versión preliminar de este artículo se presentó en las 48 ํㅜㄹeunión Nacional de Bibliotecarios (Mancini, 2016)
La investigación en Bibliotecología y Ciencia de la Información (B/CI) suele ser una problemática abordada en la literatura del área pero en escasas ocasiones problematizada por quienes ingresan a la formación profesional de las disciplinas, cuyos horizontes suelen estar motivados por una inserción profesional temprana (Vallejo Sierra, 2015). En este sentido, se encuentran mayores similitudes con la Enfermería, el Trabajo Social o las Ingenierías, donde el tiempo dedicado al ejercicio profesional sobrepasa con creces a la investigación.

Los colectivos de profesionales acostumbran a compartir sus experiencias en el área y presentar avances de investigación en eventos como Jornadas, Reuniones o Congresos. En el transcurso de una investigación enmarcada en el sistema científi$c o$, las presentaciones a eventos tienen en la mayor parte de los casos una primera instancia de evaluación externa en la figura del Comité Organizador del evento y una devolución crítica de la presentación con interacción en las preguntas de las y los colegas asistentes. La disposición dispar para constituir un espacio de debate entre quienes organizan los eventos y quienes asisten a ellos se refleja en el tiempo destinado a las preguntas y en la efectiva realización de estas. Los resultados de las ponencias constituyen gran parte del cúmulo de información de lo que se conoce como literatura gris, que más allá de la profusión de repositorios temáticos y algunas incipientes plataformas, no suelen presentarse -en función de su visibilidad ni sus referencias- desde otros documentos. Algunos de los libros (donde se publican total o parcialmente los resúmenes de ponencias) suelen estar incluidos en las bibliografías de los programas de la formación profesional. No obstante, como tipo de documento, 
en general, adolecen de la garantía de una evaluación por pares. Si bien el sistema científico impulsa la creación de Comités Evaluadores en editoriales universitarias y de contenido científico y/o profesional, subsisten los libros cuya única revisión consiste en la corrección de estilo. ${ }^{2}$

Por lo expuesto, las revistas se imponen como la manifestación textual más rigurosa. Además de contar con una estructura en la que se da cuenta de un estado del arte, se describe la metodología, los resultados y las conclusiones. En muchos casos se explicitan incluso los tiempos de la revisión por pares.

Es correcto afirmar que existe una tradición en la publicación en revistas de B/CI, ya sea desde la escritura, la edición, la evaluación o la lectura en el contexto internacional. De hecho, algunos de los tratados fundantes de la disciplina contienen artículos de revistas de Bibliotecología entre sus bibliografías. Con el aumento del volumen de las revistas de $\mathrm{B} / \mathrm{CI}$, se han conformado universos que justifican su reunión en bases de datos. Library and Information Science Abstracts (LISA) y Library and Information Science and Technology Abstacts (LISTA) constituyen claros ejemplos de esta tradición de difusión de las ciencias de la información. Sin embargo, la representación latinoamericana e hispanoparlante en ellas es ínfima. En 2009, la aplicación de un algoritmo que evalúa la "calidad" de las revistas científicas en LISA, entre los 27 títulos rescatados, no se halló representación iberoamericana (Gorbea-Portal y Ávila Uriza, 2009).

En América Latina, la base de datos coordinada por la Universidad Nacional Autónoma de México INFOBILA (Información Bibliotecológica Latinoamericana) se ha erigido como la fuente de información que cumple este propósito. Sin embargo, las dificultades en su acceso (no se puede acceder desde la I.P. de la UBA) y manipulación de los datos (no se puede descargar como base de datos) la alejan de la concreción de este objetivo.

En la dilucidación sobre qué se publica radica uno de los grandes interrogantes de la disciplina y es, a la vez, un escondrijo de sus posibles fortalezas. Diferentes representaciones sociales sobre las labores de las y los profesionales de la información operan incluso desde las mismas disciplinas pertenecientes a las ciencias de la información. Sin duda un desafío clave consiste en superar el dilema hermenéutico presentado por Hjørland (2015) y delimitar el campo de las Ciencias de la Información a priori para luego reconocer el campo en las temáticas preponderantes dentro de las mismas revistas.

El objetivo de este trabajo es caracterizar a las revistas científicas latinoamericanas, a partir de sus artículos y países de publicación, correspondientes al período 20082016. Se busca, por un lado, dar a conocer la producción bibliotecológica a las y los profesionales de la región. Por otra parte, se propicia detectar tendencias locales y perfiles de trabajo, así como áreas de vacancia, con el propósito de formar una cartografía de la situación de la disciplina.

\section{Marco teórico y antecedentes}

Las revistas científicas en general y sus artículos en particular, han sido identificados como uno de los principales vehículos de difusión del conocimiento científico de la mayoría de las disciplinas, la B/CI no es la excepción (Gómez Fuentes, 1993; Gorbea Portal, 2007; Romanos de Tiratel, 2008; Gorbea Portal y Ávila Uriza, 2009; Gorbea Portal y Piña Pozas, 2013). Es por ello que, tal como lo reflejan los trabajos de Gorbea Portal y de Martínez Comeche (2010), el análisis periódico de este tipo de literatura científica, a lo largo del tiempo y en contextos específicos, permite tener un panorama
2. Estas características también forman parte del habitus de cada colectivo disciplinar y merecen ser abordadas científicamente. 
de la disciplina, especialmente en el plano teórico: qué paradigmas la afectan y adopta o supera, cómo y con qué otras áreas del conocimiento se interrelaciona y cómo evolucionan sus conceptos (Capurro, 2007; Hjørland, 2015; Aharony, 2011). A su vez, esa sistematización del estudio de la literatura especializada genera posibilidades para el estudio de otras realidades particulares de la disciplina.

Este trabajo se enmarca en lo que se puede pensar como dos potenciales tendencias actuales en el estudio de la literatura científica en B/CI. La primera se debe al resurgimiento de los estudios bibliométricos, ahora abordados desde otras perspectivas, que permiten apreciar y evaluar -especialmente en contextos de países, regiones o instituciones de "economía y ciencia periféricas"- el desarrollo de la disciplina y relacionar otros aspectos más allá de su mero aporte a nivel internacional (Gorbea Portal y Piña Pozas, 2013). La segunda tendencia corresponde a los estudios desde la perspectiva de la organización del conocimiento, cuáles son las características de esta comunidad científica en particular (delimitada geográfica y/o institucionalmente) y con qué otras disciplinas se relaciona. Estos dos ejes de la investigación representan los aspectos prácticos y teóricos cuya articulación es esencial en la investigación bibliotecológica.

Una vez reunidos los temas que surgen de un estudio cuantitativo y, a la vista del conjunto de los mismos, se ofrece un amplio panorama de posibilidades para su análisis. Una de ellas es clasificarlos en áreas temáticas. Establecer las categorías conforme las que se generarán esas áreas temáticas es uno de los ejercicios teóricos más enriquecedores para una disciplina porque pone en discusión la actualidad de sus conceptos, sus teorías, métodos y las miradas de sus investigadores, profesionales, usuarios y estudiantes como actores claves (Capurro, 2007).

En cuanto a los antecedentes, ya se ha elaborado un estado del arte sobre estudios bibliométricos en B/CI (Arquero Avilés, 2003). La cantidad de estudios en idioma inglés exceden el propósito de este ejercicio de revisión, pero se pueden destacar los trabajos de Davarpanah y Aslekia (2008), en el que toman un núcleo de 56 revistas en idioma inglés para analizar las temáticas presentes, patrones de distribución de autores, instituciones, disposición de las citas y su obsolescencia. Cabe destacar que entre los 894 artículos relevados, la cantidad de autores pertenecientes a una Iberoamérica representada por España, Brasil, Colombia, México y Portugal oscilan entre 11 y 22.

Desde América Latina se han realizado estudios sobre las revistas de B/CI (GorbeaPortal y Ávila Uriza, 2009). Hacia fines del milenio pasado México, Brasil y Argentina se erigían como los países con mayor cantidad de revistas (Urbizagástegui Alvarado, 2006). Esta contribución es uno de los antecedentes más cercanos, tanto en cuanto a la elección del objeto de estudio como en su temporalidad. No obstante, la volatilidad del mismo objeto ocasiona que este trabajo requiera una actualización.

No se han detectado artículos en los que todos los países latinoamericanos estén representados. El texto de Gorbea-Portal y Ávila Uriza (2009) arrojó un total de 888 títulos sin distinción entre países, pero entre las que han jerarquizado según diferentes criterios formales no se encontraban títulos ni latinoamericanos ni en idioma español.

A nivel país, se ha realizado análisis de dominio en Cuba para el período 2005-2008 (Lozano Díaz, Rodríguez Sánchez y Morffe Félix, 2008), en donde los tópicos predominantes han sido Gerencia de Información (31\%), seguido de Fundamentos de la Bibliotecología y las Ciencias de la Información (23\%) y Servicios de la Información (21\%).

En Argentina se ha caracterizado la producción de la revista Referencias, editada por la Asociación de Bibliotecarios Graduados de la República Argentina (ABGRA) entre 1994 y 2002 (Liberatore, et al. 2003). El trabajo realizado en la Universidad Nacional 
de Mar del Plata sobre un análisis de dominio de la revista editada por ABGRA, contempló un recorte temático que fue construido en base a la extracción de palabras clave de los documentos y las áreas temáticas definidas en las Reuniones de Directores y Docentes de Escuelas de Bibliotecología del MERCOSUR. En los resultados arrojados, la categoría más prominente resultó ser Profesión y bibliotecas con un $45 \%$, superando a Tecnologías de la Información (15\%) y Gestión (14\%) (Liberatore, et al. 2003). La amplitud de temáticas posibles incluidas en la primera categoría, su inclusión en el área de Fundamentos Teóricos dentro del esquema del MERCOSUR y la distancia con respecto a las categorías restantes resultan datos suficientes como para considerar no establecerla como tópico a codificar.

En la Universidad Nacional de Córdoba sobre un total de 27 tesinas presentadas entre 1996 y 2012, 8 fueron sobre Procesos Técnicos y 8 sobre Gestión, siendo éstas las áreas más prominentes y superando a Fundamentos Teóricos y Recursos y Servicios (Martín, 2012).

Uno de los más recientes análisis temáticos bibliométricos se ha hecho sobre las Jornadas de Intercambio y Reflexión acerca de la Investigación en Bibliotecología (Miguel, 2016). Sobre un total de 105 ponencias las áreas predominantes han sido Estudios métricos (20,8\%), Historia del Libro y las Bibliotecas (18,8\%) y Procesos técnicos/Organización, representación y recuperación de la información (16,6\%).

En su Trabajo de Campo para la obtención de la Licenciatura en Bibliotecología y Ciencia de la Información con orientación en Procesamiento de la Información en la Universidad de Buenos Aires, Mancini $(2013,2014)$ buscó detectar la presencia de los procesos técnicos en las revistas de Bibliotecología recientes de América Latina en idioma español. Para ello se generó una base de datos en la que se intentó cuantificar la relación entre los tópicos predominantes y la escritura. Entre los resultados arrojados se verificó que los procesos técnicos están representados en menor medida que aquellos trabajos sobre recursos y servicios, gestión, tecnología y bibliometría.

\section{Metodología}

Para poder relevar la producción bibliográfica bibliotecológica latinoamericana hispanoparlante en revistas científicas, se identifican el mayor número posible de revistas de la región. En esta misma etapa, se excluyeron las revistas brasileñas y españolas. En un segundo momento, se clasifica las revistas relevadas como generales u orientadas a determinados tópicos. Los resultados arrojan una considerable presencia de estudios bibliométricos en Ciencias de la Información.

Para la selección de las revistas se consideran parámetros generales de revistas científicas, tales como existencia de resumen, periodicidad regular y bibliografía actualizada, entre otros.

Algunas revistas que pudieron conseguir aceptación en la comunidad científica, han visto modificada su URL de acceso, ocasionando un desmembramiento de la colección digital. En otros casos se valora el contenido, pero las deficiencias formales son tales que resulta difícil considerar el título para su inclusión. De los 28 títulos, en 8 ocasiones no se ha detectado referato para el período completo. Sin embargo, justamente la intención de representar la mayor cantidad posible de países, conlleva a tomar una serie de decisiones que permitan contemplar el relevamiento de algunas revistas profesionales con características de revistas científicas. Varias de estas no han tenido en cuenta mínimos criterios establecidos por los servicios de indización y resúmenes. Una de las decisiones metodológicas consistió en determinar las secciones a incluir 
3. Se utilizó Latindex, INFOBILA, los catálogos de las bibliotecas del IIBI-UNAM, el INIBI-FFyLUBA y bibliotecas universitarias y nacionales de cada país. en el relevamiento de los artículos. Se han considerado todos aquellos artículos que al menos contengan un resumen, de esta forma, se omiten reseñas, comunicaciones y notas editoriales. También se toma en cuenta que las revistas tengan contribuciones externas y una evaluación desde el Comité Editor.

Luego de una primera aproximación se ha comprobado que algunos países han editado revistas en la última década pero hoy no lo hacen. Por ello, se adoptó el período 20082016 para acotar el relevamiento y poder analizar el presente y las tendencias actuales.

A través de la búsqueda por bibliografías de publicaciones periódicas, portales de revistas y catálogos de hemerotecas, ${ }^{3}$ se identificaron los siguientes títulos: Información, Cultura y Sociedad, Infodiversidad, Palabra Clave y Anuario de la Escuela de Archivología (Argentina); Fuentes: Revista de la Biblioteca y Archivo Histórico de la Asamblea Legislativa Plurinacional (Bolivia); Infoconexión (Chile); Revista Interamericana de Bibliotecología, Códices (Colombia); Bibliotecas, E-Ciencias de la Información, Revista AIBDA (Costa Rica); ACIMED (continuada por Revista Cubana de Información en Ciencias de la Salud), Bibliotecas: Anales de Investigación, Ciencias de la Información (Cuba); Investigación Bibliotecológica, Biblioteca Universitaria, Bibliotecas y Archivos, Bibliotecología Crítica (México); Biblios, Alexandria, Infoacceso (Perú); Simbiosis, AccesBib (Puerto Rico); Informatio (Uruguay);Enl@ce y Hélice (Venezuela).

Una vez detectados estos títulos, con el fin de representar más fielmente al resto de los países, se generó un protocolo de correo para contactar a miembros de las asociaciones profesionales de cada país y así se identificó a Códice 020.9866: Revista ecuatoriana de Bibliotecología (Ecuador). Al finalizar la codificación, se identificaron las revistas chilena Ciencias de la Documentación y la argentina Prefacio. Dichas publicaciones serán incorporadas en una próxima versión de la base.

La cantidad total de revistas aporta un dato ilustrativo del desarrollo de la disciplina en la región. Hace dos décadas, tomando como fuente The Serials Directory, se detectó un total de 16 revistas científicas de la especialidad. Al añadir otras fuentes, se llegó a un total de 26 , de las cuales solo 16 eran editadas en lengua hispana (Gómez Fuentes, 1993). En esta investigación interesa verificar si la cantidad de revistas relevadas en la actualidad superará a las relevadas en aquel momento.

Una vez identificadas las revistas, una segunda etapa de este trabajo inscripto en la tradición bibliográfica consiste en relevar las temáticas de cada uno de sus artículos. La definición de los tópicos implica múltiples decisiones que llevan a considerar las fronteras internas y externas de la disciplina: ¿desarrollo de la colección debería compartir la categoría con alfabetización de la información? ¿La Archivología y la Museología se incluyen? ¿Los estudios métricos de información constituyen una categoría autónoma?

Con respecto a la codificación de los artículos, para la construcción de categorías se evaluó la posibilidad de utilizar esquemas de clasificación, tesauros, taxonomías utilizadas en otros artículos y líneas de estudio de institutos de investigación. Finalmente, se ha resuelto tomar como base las áreas en las que se trabajan los contenidos mínimos a dictarse en las carreras de B/CI del MERCOSUR por motivos pragmáticos, esta clasificación ya había sido utilizada en Mancini $(2013,2014)$.

Para el análisis de las temáticas, se ha construido una base de datos donde se han relevado los siguientes campos: país, título de la revista, año, volumen, número, número de artículo dentro de cada ejemplar, título del artículo, resumen, palabras clave, sección dentro de la revista, filiación institucional de los autores, clase principal y hasta dos clases secundarias. Se creó la categoría de clases secundarias para 
representar, de forma ponderada, la múltiple pertenencia a áreas temáticas. Este rasgo es común en numerosos casos. No se discriminará el país de origen de los autores para esta etapa de la investigación.

\section{Resultados}

El relevamiento detallado anteriormente arrojó un total de 28 revistas latinoamericanas de Bibliotecología que se han editado en español entre 2008 y 2016. Sumando las dos revistas que no fueron incluidas en esta versión de la base, eso da un total de 30. Se trata una cifra superior aunque similar a las relevadas por Gómez Fuentes (1993). La impresión es que no hay una acumulación progresiva de revistas, ni de artículos en el período señalado. Por ejemplo, el número de artículos por año no presenta tendencia positiva alguna sino que fluctúa entre 220 (2016) y 295 (2012), con un promedio de 265 artículos por año.

\begin{tabular}{|c|c|c|c|c|c|c|c|c|c|c|c|c|}
\hline \multirow[t]{2}{*}{ Revista } & \multirow[t]{2}{*}{ País } & \multicolumn{9}{|c|}{ Año de publicación } & \multirow[t]{2}{*}{ Total } & \multirow[t]{2}{*}{$\%$} \\
\hline & & 2008 & 2009 & 2010 & 2011 & 2012 & 2013 & 2014 & 2015 & 2016 & & \\
\hline Infodiversidad & Argentina & 6 & 5 & 9 & 5 & 5 & - & - & - & - & 30 & 1 \\
\hline Palabra Clave & Argentina & - & - & - & 3 & 8 & 5 & 7 & 8 & 9 & 40 & 2 \\
\hline $\begin{array}{l}\text { Anuario Escuela de } \\
\text { Archivología }\end{array}$ & Argentina & - & 6 & 11 & 13 & 13 & - & 11 & - & - & 54 & 2 \\
\hline $\begin{array}{l}\text { Información, cultura y } \\
\text { sociedad }\end{array}$ & Argentina & 10 & 11 & 9 & 14 & 10 & 13 & 15 & 16 & 17 & 115 & 5 \\
\hline Bibliotecología Crítica & México & 8 & 7 & 10 & 7 & 7 & 4 & 9 & 9 & - & 61 & 3 \\
\hline Biblioteca Universitaria & México & 7 & 8 & 12 & 10 & 8 & 8 & 8 & 10 & 6 & 77 & 3 \\
\hline Bibliotecas y archivos & México & - & - & - & - & - & 4 & 8 & 8 & - & 20 & 1 \\
\hline $\begin{array}{l}\text { Investigación } \\
\text { Bibliotecológica }\end{array}$ & México & 24 & 24 & 24 & 24 & 24 & 24 & 24 & 24 & 28 & 220 & 9 \\
\hline $\begin{array}{l}\text { Bibliotecas. Anales de } \\
\text { Investigación }\end{array}$ & Cuba & 5 & 5 & 7 & 7 & - & 18 & 20 & 16 & 18 & 96 & 4 \\
\hline $\begin{array}{l}\text { ACIMED/Revista } \\
\text { Cubana de Información } \\
\text { en Ciencias de la Salud }\end{array}$ & Cuba & 69 & 55 & 22 & 23 & 30 & 33 & 28 & 30 & 36 & 326 & 14 \\
\hline $\begin{array}{l}\text { Ciencias de la } \\
\text { Información }\end{array}$ & Cuba & 21 & 20 & 30 & 26 & 30 & 22 & 21 & 24 & 4 & 198 & 8 \\
\hline Infoacceso & Perú & - & - & - & - & - & - & 11 & 2 & - & 13 & 1 \\
\hline $\begin{array}{l}\text { Alexandría: Revista } \\
\text { de Ciencias de la } \\
\text { Información }\end{array}$ & Perú & - & - & 5 & 4 & 5 & - & - & - & - & 14 & 1 \\
\hline Biblios & Perú & 30 & 25 & 0 & 23 & 26 & 31 & 25 & 24 & 24 & 208 & 9 \\
\hline \multicolumn{2}{|c|}{ Subtotal cuatro países principales } & 180 & 166 & 139 & 159 & 166 & 162 & 187 & 171 & 142 & 1472 & 62 \\
\hline \multicolumn{2}{|l|}{ Total base } & 259 & 252 & 234 & 286 & 295 & 277 & 285 & 280 & 220 & 2388 & 100 \\
\hline
\end{tabular}

Tabla 1A. Distribución de artículos según revista y año de publicación. Subtotal cuatro países principales. 


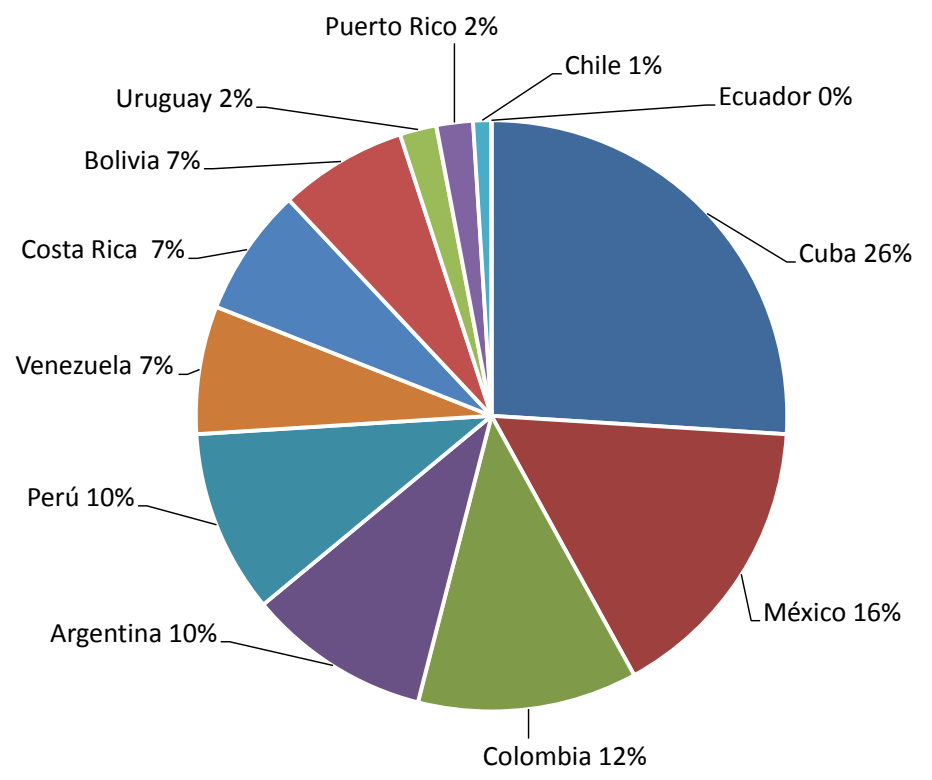

En cuanto a la distribución de los artículos por revista y año, en la Tabla 1A, se observa que 5 revistas concentran el $45 \%$ de los artículos. Se trata de las revistas cubanas de ACIMED/Revista Cubana de Información en Ciencias de la Salud (14\%) y Ciencias de la Información (8\%), la mexicana Investigación Bibliotecológica (9\%), la peruana Biblios (9\%) y la argentina Información, Cultura y Sociedad (5\%). Estas revistas presentan artículos en todos los años del período, característica que comparten con Bibliotecología Crítica (excepto el año 2016) y con la cubana Bibliotecas:Anales de Investigación (excepto el año 2012). El resto de las 6 revistas presentadas en la tabla $1 \mathrm{~A}$ comienzan en el período relevado (como Palabra Clave), o bien terminan (Infodiversidad), o presentan artículos de manera no continua. Los 14 títulos incluidos en esta tabla contienen al $62 \%$ de los artículos del total de la base.

En relación al país de publicación de los artículos de la base, se observa una estructura concentrada en el Gráfico 1. En primer lugar se ubica Cuba, con el $26 \%$ de los artículos (620 de un total de 2388), en segundo lugar México (16\%) y en tercer lugar Colombia (12\%). Le siguen en cuarto y quinto puesto Argentina y Perú, 10\% cada país. Estos cinco países encabezan el ranking, y suman el 73\% de los artículos. El resto de los artículos fueron publicados por revistas de Venezuela, Costa Rica y Bolivia (7\% cada país), con una participación menor de Uruguay, Puerto Rico, Chile y Ecuador.

Entre las 13 revistas que reúnen al 38\% restante de los artículos (Tabla 1B), se destacan la colombiana Revista Interamericana de Bibliotecología (7\% del total), la boliviana Fuentes: Revista de la Biblioteca y Archivo Histórico de la Asamblea Legislativa Plurinacional (7\%) y la venezolana Enl@ce: Revista Venezolana de Información,Tecnología y Conocimiento (6\%). Las tres revistas suman el $20 \%$ del total, casi la mitad del subgrupo. Las colombiana y venezolana mencionadas, junto con la costarricense Bibliotecas, son las únicas revistas de este conjunto en presentar artículos en todos los años relevados por la base. Fuera de estas 4 revistas, se encuentran 9 títulos que absorben el $13 \%$ restante de artículos. 


\begin{tabular}{|c|c|c|c|c|c|c|c|c|c|c|c|c|}
\hline \multirow{2}{*}{ Revista } & \multirow{2}{*}{ País } & \multicolumn{9}{|c|}{ Año de publicación } & \multirow{2}{*}{ Total } & \multirow{2}{*}{$\%$} \\
\hline & & 2008 & 2009 & 2010 & 2011 & 2012 & 2013 & 2014 & 2015 & 2016 & & \\
\hline E-Ciencias de la información & Costa Rica & - & - & - & 14 & 15 & 15 & 11 & 9 & 9 & 73 & 3 \\
\hline Revista AIBDA & Costa Rica & 6 & 7 & - & - & - & - & - & - & - & 13 & 1 \\
\hline Bibliotecas & Costa Rica & 13 & 8 & 9 & 10 & 12 & 8 & 7 & 8 & 8 & 83 & 3 \\
\hline $\begin{array}{l}\text { Códices: Revista de la } \\
\text { Facultad de Ciencias } \\
\text { Económicas y Sociales: } \\
\text { Programa de Sistemas de } \\
\text { Información y Documentación }\end{array}$ & Colombia & 14 & 14 & 14 & 13 & 12 & 13 & 13 & 12 & - & 105 & 4 \\
\hline $\begin{array}{l}\text { Revista Interamericana de } \\
\text { Bibliotecología }\end{array}$ & Colombia & 16 & 23 & 20 & 23 & 25 & 16 & 16 & 16 & 18 & 173 & 7 \\
\hline AccesBib & Puerto Rico & - & - & - & - & 5 & 12 & - & 3 & - & 20 & 1 \\
\hline Simbiosis & Puerto Rico & - & 5 & 8 & 3 & 10 & - & - & - & - & 26 & 1 \\
\hline $\begin{array}{l}\text { Hélice: Revista Venezolana en } \\
\text { Ciencias de Información }\end{array}$ & Venezuela & - & 10 & 10 & - & - & - & - & - & - & 20 & 1 \\
\hline $\begin{array}{l}\text { Enl@ce: Revista Venezolana } \\
\text { de Información, Tecnología y } \\
\text { Conocimiento }\end{array}$ & Venezuela & 19 & 16 & 16 & 15 & 17 & 19 & 18 & 18 & 12 & 150 & 6 \\
\hline $\begin{array}{l}\text { Fuentes, Revista de la } \\
\text { Biblioteca y Archivo Histórico } \\
\text { de la Asamblea Legislativa } \\
\text { Plurinacional }\end{array}$ & Bolivia & - & 3 & 11 & 30 & 23 & 24 & 25 & 27 & 23 & 166 & 7 \\
\hline $\begin{array}{l}\text { Infoconexión: Revista Chilena } \\
\text { de Bibliotecología y Gestión } \\
\text { de Información }\end{array}$ & Chile & - & - & 7 & 10 & 6 & - & - & - & - & 23 & 1 \\
\hline $\begin{array}{l}\text { Códice 020.9866: } \\
\text { Revista Ecuatoriana de } \\
\text { Bibliotecología }\end{array}$ & Ecuador & - & - & - & - & - & - & - & 8 & - & 8 & 0 \\
\hline Informatio & Uruguay & 11 & - & - & 9 & 4 & 8 & 8 & 8 & 8 & 56 & 2 \\
\hline \multicolumn{2}{|c|}{ Subtotal resto de países } & 60 & 71 & 86 & 103 & 102 & 92 & 80 & 92 & 61 & 747 & 31 \\
\hline \multicolumn{2}{|l|}{ Total base } & 259 & 252 & 234 & 286 & 295 & 277 & 285 & 280 & 220 & 2388 & 100 \\
\hline
\end{tabular}

Tabla 1B. Distribución de artículos según revista y año de publicación. Resto de países.

Se agrupa luego a los países en base a la cantidad de revistas, artículos y periodicidad, por un lado aquellos que han editado más de una revista en el período y/o superan el promedio de 10 artículos por año entre sus títulos o editan más de un número por año (Argentina, México, Cuba, Colombia, Costa Rica y Perú) y, por otro lado, aquellos que no alcanzan estos requisitos (Bolivia, Chile, Ecuador, Puerto Rico y Uruguay). Se presume que Chile podría pertenecer al primer grupo si se añade Ciencias de la Documentación y se destaca, por otra parte, la cantidad de contribuciones logradas por la revista boliviana Fuentes.

En la Tabla 2, de acuerdo a la clasificación realizada de los artículos, se observa que dos categorías (Teoría, Historia, Educación e Investigación y Recursos y Servicios) concentran una proporción importante de casos según la categoría principal, con el $26 \%$ y $18 \%$ respectivamente, casi la mitad de la base. Le siguen 
Tabla 2. Distribución de clases principal y clases secundarias

4. Tienen más de un tercio de sus artículos en esta categorías varias otras revistas: Simbiosis, Información, cultura y sociedad,

Hélice: Revista Venezolana en Ciencias de Información, Revista Interamericana de Bibliotecología, Bibliotecas, Informatio, Biblioteca Universitaria, Bibliotecas. Anales de Investigación y Palabra Clave.

5. Procesos técnicos, Recursos y Servicios, Gestión y Preservación son agrupados como Actividades Profesionales; Teoría, Historia, Educación e Investigación y Estudios métricos bajo Estudios teóricos y métricos y Tecnología de la Información, Archivología se subsumen a las Otras disciplinas. en importancia Gestión, Otras disciplinas y Estudios Métricos (12\%, 11\% y 10\% respectivamente, un tercio de la base). Completan la distribución Archivología (9\%), Tecnología de la información (8\%), Procesos técnicos (5\%) y Preservación (1\%). Al incorporar las dos clases secundarias a la distribución, la base aumenta un $35 \%$, y se observa solo cambios menores que no afectan a la distribución en forma significativa.

\begin{tabular}{|c|c|c|}
\hline Clase & Principal & $\begin{array}{l}\text { Principal y } \\
\text { secundarias }\end{array}$ \\
\hline Procesos técnicos & 5 & 6 \\
\hline Recursos y servicios & 18 & 18 \\
\hline Gestión & 12 & 12 \\
\hline Tecnología de la información & 8 & 11 \\
\hline Archivología & 9 & 8 \\
\hline Preservación & 1 & 1 \\
\hline Teoría, Historia, Educación e Investigación & 26 & 24 \\
\hline Otras disciplinas & 11 & 11 \\
\hline Estudios métricos & 10 & 9 \\
\hline \multirow{2}{*}{ Total } & 100 & 100 \\
\hline & 2388 & 3233 \\
\hline
\end{tabular}

Fuente: elaboración propia

Con respecto al análisis del perfil de cada revista según la clasificación propuesta dentro de la clase principal, no se presenta un cuadro que detalle esta información para cada revista dado el tamaño del mismo. Interesa, sin embargo, destacar algunos hallazgos al respecto. Ninguna revista tiene el $25 \%$ o más de sus artículos sobre Procesos técnicos, Gestión y Preservación. Sólo Códice 020.9866: Revista Ecuatoriana de Bibliotecología tiene $25 \%$ de sus artículos sobre Tecnología de la información. Ahora bien, en relación con Recursos y Servicios, Infoconexión posee el $43 \%$ de sus artículos sobre este tema y AccesBib el 35\%. El caso de Archivología es particular, ya que Anuario Escuela de Archivología presenta el $91 \%$ de sus artículos sobre esta disciplina, y consiste en la única revista claramente especializada de la base. En cuanto a la Teoría, Historia, Educación e Investigación encontramos tres revistas con la mitad o más de artículos en esta categoría: Bibliotecología Crítica (69\%), Infodiversidad (67\%) y Códice 020.9866: Revista Ecuatoriana de Bibliotecología (50\%). ${ }^{4} \mathrm{Al}$ tratarse de una categoría amplia, no es posible especificar si se identifica con revistas especializadas o no, aunque se infiere que la Bibliotecología Social es el tema preponderante en Bibliotecología Crítica y los estudios históricos lo son en Infodiversidad. Interesa destacar que Fuentes y Enl@ce tienen un 49\% y $36 \%$ de artículos de otras disciplinas respectivamente, donde la historia de la disciplina prepondera en el primer caso y las ciencias de la comunicación en el segundo. Por último, cabe destacar que un $46 \%$ de los artículos de Revista AIBDA son sobre Estudios Métricos.

Un análisis de la clase principal agrupada con las secundarias se presenta a partir del Gráfico 2 con el fin de simplificar el análisis. Se presentan las revistas según su participación ordenada de menor a mayor en actividades profesionales 5 .

Infoconexión aparece con el 70\% de sus artículos de actividades profesionales, seguida por AccesBib (50\%). Se destacan en los estudios téoricos y métricos sobre la profesión Bibliotecología Crítica (70\%), Infodiversidad (67\%), Información, Cultura y 
$\square$ Actividades profesionales

$\square$ Estudios teóricos y métricos

$\square$ Otras disciplinas

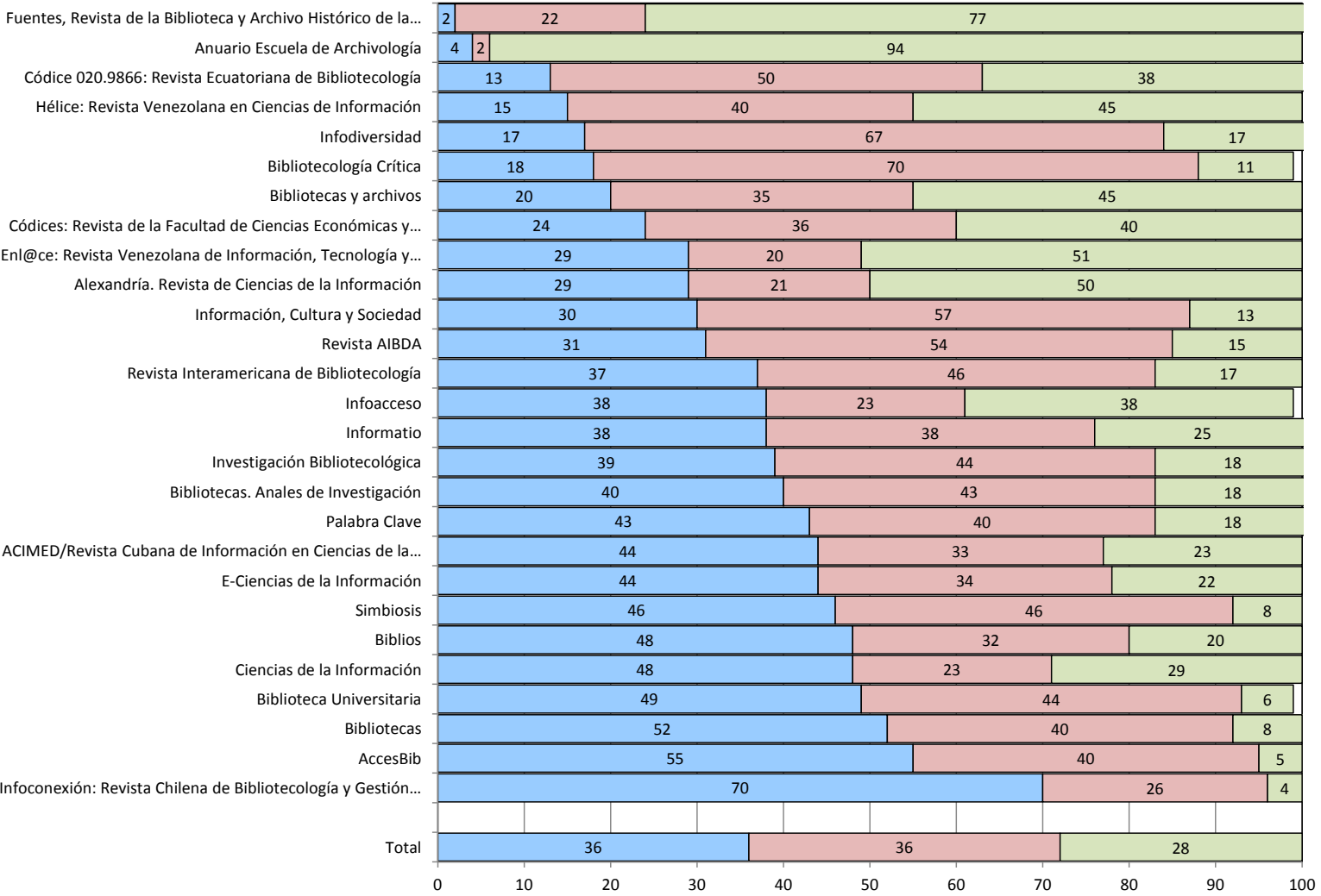

Sociedad (57\%), Revista AIBDA (54\%) y Códice 020.9866 (50\%). Con respecto a las otras disciplinas vuelven a sobresalir Anuario Escuela de Archivología (ahora con 94\%), Fuentes (77\%),Enl@ce (51\%) y Alexandrí (50\%). A partir del análisis agrupado se refuerzan elementos observados en el análisis desagregado de las revistas (pocas revistas especializadas, fuerte presencia de estudio teóricos y métricos y de actividades profesionales), a la vez que se arroja luz sobre los perfiles de las revistas, especialmente las mencionadas en este párrafo (Bibliotecología Crítica e Infodiversidad con estudios teóricos y métricos, y Fuentes con otras disciplinas).

Es de especial interés para los objetivos del artículo la distribución por países de las revistas de la base, información presentada en el Gráfico 3.

De un total de 27 revistas, se observa a Argentina y México con el $15 \%$ cada uno (4 revistas), seguidas de Costa Rica, Cuba y Perú con el $11 \%$ por país (3 revistas). Estos cinco países suman casi dos tercios del total (63\%). El tercio restante se compone de Colombia, Puerto Rico y Venezuela ( $7 \%$ cada país, con 2 revistas por país) y Bolivia, Chile, Ecuador y Uruguay con ( $4 \%$ cada país, con una revista por país). Se trata de una distribución relativamente concentrada en unos pocos países, con particular presencia de México y Argentina, ambos con los principales institutos de investigación sobre Bibliotecología en la América Latina de habla hispana. 
Gráfico 3. Distribución de revistas por países de publicación

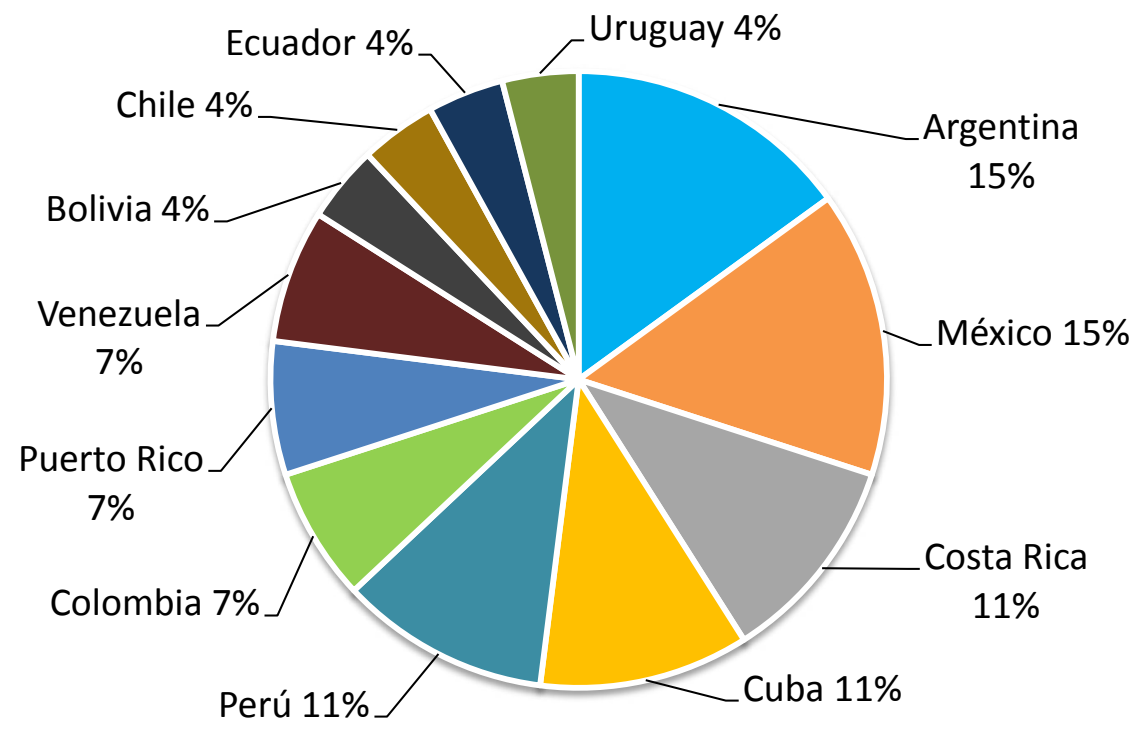

Fuente: elaboración propia

Para concluir el análisis se describen los perfiles de áreas temáticas por país (Tablas 3 y 4). Se excluyen los que cuentan con una sola revista, para evitar confundir los tipos de análisis.

\begin{tabular}{|c|c|c|c|c|c|c|c|c|}
\hline \multirow[b]{2}{*}{ Clase principal } & \multicolumn{8}{|c|}{ País de publicación } \\
\hline & Argentina & Colombia & Costa Rica & Cuba & México & Perú & $\begin{array}{l}\text { Puerto } \\
\text { Rico }\end{array}$ & Venezuela \\
\hline Procesos técnicos & 7 & 6 & 5 & 5 & 6 & 7 & 4 & 2 \\
\hline Recursos y servicios & 13 & 16 & 26 & 19 & 19 & 23 & 33 & 14 \\
\hline Gestión & 3 & 8 & 14 & 20 & 10 & 14 & 13 & 11 \\
\hline $\begin{array}{r}\text { Tecnología de la } \\
\text { información }\end{array}$ & 4 & 3 & 9 & 11 & 8 & 9 & 7 & 14 \\
\hline Archivología & 23 & 19 & $\mathrm{o}$ & 4 & 4 & 8 & o & 2 \\
\hline Preservación & 1 & 2 & 2 & 1 & 1 & 3 & o & o \\
\hline $\begin{array}{r}\text { Teoría, Historia, Educación } \\
\text { e Investigación }\end{array}$ & 36 & 34 & 31 & 15 & 34 & 20 & 39 & 21 \\
\hline Otras disciplinas & 5 & 4 & 6 & 8 & 4 & 6 & $\mathrm{o}$ & 34 \\
\hline Estudios métricos & 7 & 8 & 8 & 17 & 14 & 11 & 4 & 2 \\
\hline Total & $\begin{array}{l}100 \\
239\end{array}$ & $\begin{array}{l}100 \\
278\end{array}$ & $\begin{array}{l}100 \\
169\end{array}$ & $\begin{array}{l}100 \\
620\end{array}$ & $\begin{array}{l}100 \\
378\end{array}$ & $\begin{array}{l}100 \\
235\end{array}$ & $\begin{array}{l}100 \\
46\end{array}$ & $\begin{array}{l}100 \\
170\end{array}$ \\
\hline
\end{tabular}


En Tabla 3 se registra en primer lugar una distribución similar en los países seleccionados en torno a la concentración de casos alrededor de las principales clases: Recursos y servicios y Gestión (incluidos como Actividades Profesionales) y Teoría, Historia, Educación e Investigación (como estudios teóricos y métricos). Esto puede interpretarse como un primer indicio de no especialización entre países.

\begin{tabular}{|c|c|}
\hline País & $\begin{array}{c}\text { Actividades } \\
\text { profesionales }\end{array}$ \\
\hline Puerto Rico & 50 \\
\hline Costa Rica & 47 \\
\hline Perú & 46 \\
\hline Cuba & 45 \\
\hline México & 37 \\
\hline Total & 36 \\
\hline Colombia & 32 \\
\hline Venezuela & 27 \\
\hline Argentina & 25 \\
\hline
\end{tabular}

\begin{tabular}{|c|c|}
\hline País & $\begin{array}{c}\text { Estudios } \\
\text { teóricos } \mathbf{y} \\
\text { métricos }\end{array}$ \\
\hline México & 48 \\
\hline Puerto Rico & 43 \\
\hline Argentina & 43 \\
\hline Colombia & 42 \\
\hline Costa Rica & 38 \\
\hline Total & 36 \\
\hline Cuba & 32 \\
\hline Perú & 31 \\
\hline Venezuela & 22 \\
\hline
\end{tabular}

\begin{tabular}{|c|c|}
\hline País & $\begin{array}{c}\text { Otras } \\
\text { disciplinas }\end{array}$ \\
\hline Venezuela & 51 \\
\hline Argentina & 33 \\
\hline Total & 28 \\
\hline Colombia & 26 \\
\hline Cuba & 24 \\
\hline Perú & 23 \\
\hline México & 16 \\
\hline Costa Rica & 15 \\
\hline Puerto Rico & 7 \\
\hline
\end{tabular}

Tabla 4. Distribución de clase principal agrupada según países de publicación seleccionados

En una segunda lectura, un tercio de los artículos de Puerto Rico se ubican en la clase Recursos y servicios, $20 \%$ de los de Cuba en Gestión, $14 \%$ de los de Venezuela en Tecnología de la información, $23 \%$ de los artículos de Argentina y 19\% de los de Colombia en Archivología, 34\% de los de Venezuela en Otras disciplinas y 17 de los de Cuba en Estudios métricos. Si bien no se puede hablar de perfiles especializados en alguna de las clases señaladas, hay diferencias interesantes entre los países. Al agrupar las clases (Tabla 4), se intenta analizar si hay países que se ubiquen en alguna situación particular. Para ello se calcula el desvío estándar de cada columna, y se señalan los países que se ubican a más de un desvío estándar del total. Se encuentra así que Puerto Rico, Costa Rica, Perú y Cuba tienen más artículos sobre el núcleo disciplinar que el promedio, mientras que Argentina menos. En cuanto a los estudios sobre la profesión, México se encuentra por sobre el total y Venezuela debajo. Por último, en cuanto a las Otras disciplinas, Venezuela presenta más artículos que el porcentaje del total, mientras que Puerto Rico y Costa Rica se ubican por debajo.

\section{Reflexiones finales}

Se considera que la producción latinoamericana conforma un escenario de producción estable sin tendencia clara de crecimiento, con un promedio de 265 artículos por año. Esta estabilidad quizás esconda cambios en la distribución interna de esa producción, escenario verificable a partir de un análisis longitudinal de las clases principales.

Tanto en los países de publicación de las revistas como en la distribución de los artículos por revista, se expresa una distribución concentrada en pocos casos $(3$ a 5) y con un conjunto de países y revistas subrepresentados, especialmente en el caso de la cantidad de artículos por país. Se resalta que mientras hay países con una práctica constante en la publicación de revistas científicas (Cuba, México, Argentina, y Colombia), coexiste una periferia con producción no sistematizada en revistas (la mayor parte de países del Caribe y América Central y Paraguay). Aquellos 
países con tradición teórica e institucional en América Latina como México con el IIBI (ex CUIB) de la UNAM, Argentina con el INIBI de la UBA y Colombia con la Escuela Interamericana de Bibliotecología no sólo han logrado que las revistas editadas por estas instituciones se consoliden, sino que además han propiciado la creación de otros títulos. La futura inclusión de la revista Prefacio, editada por la Universidad Nacional de Córdoba, se inscribe en esta línea.

Con respecto a las áreas temáticas, en términos absolutos las revistas que más artículos poseen sobre Teoría, Historia, Enseñanza e Investigación son la Revista Interamericana de Bibliotecología (61), Investigación Bibliotecológica (53) e Información, Cultura y Sociedad (51). Este dato evidencia que la tradición teórica de los centros de investigación se corresponde también con cierto perfil temático de sus revistas.

Merece un análisis excepcional un título. Se trata de la revista cubana ACIMED, continuada como Revista Cubana de Información en Ciencias de la Salud. Una de las posibles razones de su destacada producción (326 artículos, $14 \%$ de la base) probablemente sea la vitalidad de las ciencias de la salud como actividad científica y profesional en la isla caribeña. De corroborarse este tipo de sinergia entre la Bibliotecología y otras disciplinas podría ser un modelo interesante para impulsar la producción en la región.

Son pocas revistas especializadas o con presencia destacada de alguna temática. Si en las revistas es posible hablar de especialización, ¿sucede lo mismo con los países?, ¿o se trata de una concentración de casos sin una pauta direccionada de desarrollo en torno a algunas líneas prioritarias de trabajo e investigación? Se considera que no hay un escenario de especialización entre países. Aquellos con varias revistas tienden a tener un perfil similar al global de la base. De todas formas, esto no significa que no haya en términos relativos mayores concentraciones de las clases principales. Sucede que Puerto Rico, Costa Rica, Perú y Cuba tienen más artículos sobre las actividades profesionales, México sobre los estudios teóricos y Venezuela presenta más artículos sobre otras disciplinas, dado que Enl@ce es una revista que comparte desde su alcance las contribuciones bibliotecológicas con las de ciencias de la comunicación.

La complejidad de este análisis puede incrementarse si se establece que las revistas no publican exclusivamente contribuciones de autores del mismo país. Existe un antecedente en esta línea en el que se ha analizado la producción de autores de México en revistas mexicanas, pero con una reducida cantidad de casos (Clausó García, 2011). Se estima que a nivel latinoamericano dicho análisis puede ser provechoso además para detectar el grado de internacionalización de las revistas.

La perspectiva de ampliación de la investigación es extensa. En primer lugar, surge la necesidad, dada la colaboración de Brasil, Portugal y España con los países de la base (De Filippo, De Souza y Sanz Casado, 2016), de incluirlos y comparar sus tendencias temáticas con el resto de América Latina. Esta ampliación ayudará a caracterizar de manera cabal el escenario particular de América del Sur. En segundo lugar, es preciso extender la base hacia los años más recientes (2017 y 2018) así como hacia el resto del Siglo XXI (2000-2007).

Además, otro de los futuros objetos a indagar es la incorporación de otros sistemas de categorización. Esto no sólo permitiría la comparación cruzada de perfiles, sino también abrir categorías que en el estado actual de la base acumulan muchos casos como "Otras disciplinas" y "Teoría, historia, educación e investigación". A través del análisis de las citas, un trabajo detectó que las disciplinas más citadas en una selección de Core Journals entre 1996 y 2004 fueron la Ergonomía, Ciencias de la Computación y Tecnología (clasificada como categoría aparte en la clasificación inicial), Administración (clasificada como Gestión en las categorías iniciales), Comunicación y Geografía 
(Odell y Gabbard, 2008). Por último, quedan pendientes, de cara a la publicación de la base en formato CSV, una serie de tareas de consistencia, estructuración y limpieza de los datos y campos de la base de datos.

El resultado de este ejercicio además, tiene la potencialidad de generar herramientas para la construcción de lenguajes documentales. Las categorías, si bien no son un tema recurrente en la literatura referida a la organización del conocimiento, y menos en la de la B/CI, es un tópico que ha formado parte de la disciplina desde sus inicios (Barité Roqueta, 1999; Hjørland, 2015). Las categorizaciones son útiles y necesarias para todas las organizaciones, es decir no es necesario usar todas las categorías existentes para construir un lenguaje documental (Barité Roqueta, 1999; Martínez Tamayo y otros, 2004).

Asimismo, ya que el usuario de los lenguajes documentales juega un rol eminentemente activo, es fundamental no perder de vista que esa actividad procede no sólo de su conciencia o de sus "modelos mentales", sino que sus conocimientos e intereses se encuentran entrelazados en la red social y pragmática que los sustentan desde el inicio (Capurro, 2007). En el hemisferio norte se han producido categorizaciones desde marcos teóricos como la Bibliotecología basada en la evidencia (Koufogiannakis, 2004) o abordajes bottom up (Zins, 2007) En esta línea, resulta desafiante identificar a la Bibliotecología latinoamericana como un contexto con características propias que requiere de un uso particular de las categorías existentes.

La tensión entre una disciplina en el proceso de consolidación de sus revistas y otras disciplinas conexas puede ser superada a través de la transdisciplinariedad, pero desde un espacio con la solidez teórica suficiente para establecer estos puentes de forma eficaz. Los datos relevados en esta investigación constituyen un insumo en sí mismo, pero sobre todo a través de futuros desarrollos tiene el potencial de, en las palabras de Sandra Miguel, mirarnos a nosotros mismos. 


\section{Q Referencias bibliográficas}

》Aharony, Noa. 2011. Library and Information Science research areas: a content analysis of articles from the top 10 journals 2007-8. En Journal of Librarianship and Information Science. Vol. 44, no. 2, 27-35.

"Arquero Avilés, Rosario. 2003. Estudios cuantitativos y de producción, autoría y/o citación sobre análisis de la investigación en Biblioteconomía y Documentación: estado de la cuestión. En Documentación de las Ciencias de la Información. No. 26, 99-123. <http://revistas.ucm.es/index.php/DCIN/article/view/DClNo303110099A/19345> [Consulta: 5 abril 2016]

» Barité Roqueta, Mario Guido. 1999. La noción de "categoría” y sus implicaciones en la construcción y evaluación de lenguajes documentales. En Actas del IV Congreso ISKO- España. Granada: ISKO. p. 39-45

" Capurro, Rafael. 2007. Epistemología y ciencia de la información. En Enlace. Vol. 4, no. 1, 11-29. <http://www.scielo.org.ve/scielo.php?script=sci arttext\&pid=S1690-75152007000100002\&lng=es\&tlng=es $>$ [Consulta: 6 abril 2016].

"Clausó García, Adelina y Antonio Carpallo Bautista. 2011. Estudio de la producción científica de las publicaciones en México referentes a la catalogación de documentos: 1990-2009. En Revista general de infomación y documentación. Vol. 21, 127-150. <http://revistas.ucm.es/index.php/RGID/article/ view/37833/36609> [Consulta: 30 octubre 2018]

»Davarpanah, M. R. y S. Aslekia. 2008. A scientometric analysis of international LIS journals: productivity and characteristics. En Scientometrics. Vol. 77, no. 1, 21-39.

»De Filippo, Daniela; Claudia Daniele de Souza y Elías Sanz Casado. 2016. ¿La proximidad geográfica y las raíces culturales juegan un papel importante en la colaboración científica?: el caso de España, Portugal y Brasil en el área de sostenibilidad y eficiencia energética. $21 \mathrm{p}$. Trabajo presentado al $X$ Encuentro de la Asociación de Educación e Investigación en Ciencia de la Información de Iberoamérica y el Caribe, realizado en Belo Horizonte del 21 al 23 de septiembre de 2016.

» Gómez Fuentes, Héctor. 1993. Las revistas latinoamericanas de Bibliotecología y Ciencias de la Información bajo el prisma de los servicios bibliográficos internacionales. En Investigación Bibliotecológica. Vol. 7, no. 14, 27-32. <http://dx.doi. org/10.22201/iibi.0187358xp.1993.14.3815>.

»Gorbea Portal, Salvador y Ma. Maricela Piña Pozas. 2013. Propuesta de un indicador para medir el comportamiento del desarrollo disciplinar de las Ciencias Bibliotecológica y de la Información en instituciones académicas. En Investigación Bibliotecológica. Vol. 27, no. 6o, 153-18o. <http://dx.doi.org/10.1016/So187$358 \times(13) 72547-4>$.

»Gorbea Portal, Salvador y Manuel Ávila Uriza. 2009. Publicaciones seriadas en ciencias bibliotecológica y de la información: su estado actual. En Investigación Bibliotecológica. Vol. 23, no. 48, 179-209

" Gorbea Portal, Salvador. 2007. Principales revistas latinoamericanas en ciencias bibliotecológicas y de la información: su difusión y su concentración temática y geográfica. En Investigación Bibliotecológica. Vol. 21, no. 42, 79-108 
» Hjørland, Birger. 2015. Theoretical development of information science: a brief history. <http://research.ku.dk/search/?pure=en/publications/theoreticaldevelopment-of-information-science-a-brief-history\%28b94a5382-3c13455f-8417-8a2bo24af4b9\%29.html> [Consulta: 6 abril 2016].

»Koufogiannakis, Denise; Linda Slater y Ellen Crumley. 2004. A content analysis of librarianship research. En Journal of Information Science. Vol. 30 , no. 3, 227-239

»Liberatore, Gustavo; Marcela Coringrato y Adrián Amerio. 2003. Estudio de la producción profesional en Bibliotecología en la Argentina: análisis de dominio de la revista Referencias. En TransInformação. Vol. 15, no. 2, 221-229. <http://dx.doi.org/10.1590/S0103-37862003000200010>.

"Lozano Díaz, Ibis; Yaniris Rodríguez Sánchez y Mariemi Morffe Félix. 2008. Comportamiento de la Bibliotecología y las ciencias de la información en Cuba en el período 2005-2008. En ACIMED. Vol. 18, no. 6. <http://scielo.sld. $\mathrm{cu} / \mathrm{pdf} / \mathrm{aci} / \mathrm{v} 18 \mathrm{n6} / \mathrm{aci071208.pdf>}$ [Consulta: 23 marzo 2016]

»Mancini, Ignacio. 2013. Los procesos técnicos en las revistas latinoamericanas de Bibliotecología de lengua castellana (2008-2012). 18 p. Trabajo presentado al I Encuentro Latinoamericano de Estudiantes de Bibliotecología, realizado en Valparaíso del 11 al 12 de diciembre de 2013. [Conferencia inaugural]

"Mancini, Ignacio. 2014. Los procesos técnicos en las revistas latinoamericanas de Bibliotecología de lengua castellana (2008-2012). Buenos Aires: Universidad de Buenos Aires. Trabajo de Campo para la Licenciatura en Bibliotecología y Ciencia de la Información. 22 p.

» Mancini, Ignacio; Manuel Riveiro, Mariano Barberán Zangaro, Mariano Corujo, Sebastián González, Ivalú Ramírez Ibarra y Verónica Saquilán. 2016. Representación de áreas temáticas en las revistas latinoamericanas de Bibliotecología de lengua castellana (2008-2012), 11 p. Trabajo presentado a la 48를 Reunión Nacional de Bibliotecarios, realizadas en Buenos Aires del 19 al 21 de abril de 2016. <http://www. abgra.org.ar/documentos/48RNB_20160421_16oo-Ponencia.pdfs [Consulta: 30 octubre 2018]

" Martín, Sandra Gisela. 2012. Las tesinas de licenciatura en Bibliotecología y documentación de la Universidad Nacional de Córdoba, Argentina 1996-2012. En Revista Interamericana de Bibliotecología. Vol. 35, no. 3, 275-284. <http://eprints.rclis. org/20062/1/art\%203.pdf> [Consulta: 23 marzo 2016]

" Martínez Tamayo, Ana, et al. 2004. Las categorías o facetas fundamentales: una metodología para el diseño de taxonomías corporativas de sitios Web argentinos. En Ciência da Informação. Vol. 33, no. 2, 106-111. <http://revista.ibict.br/ciinf/article/ view/1052> [Consulta: 22 octubre 2018]

» Martínez-Comeche, J. A.; M. J. García Martín, J. Horcas Pulido, M. de la Peña Martín y R. I. Pérez Donoso. 2010. Semiperiodo de la literatura científica en Biblioteconomía y Documentación y la influencia de los aspectos temáticos. En Investigación Bibliotecológica. Vol. 24, no. 51, 195-207. <http://www.scielo.org.mx/pdf/ib/v24n51/ v24n51a9.pdf> [Consulta: 23 marzo 2016]

"Miguel, Sandra. 2016. Mirarnos a nosotros mismos: consolidando espacios para el debate y la reflexión sobre la investigación en Bibliotecología y Ciencia de la Información. 9 p. Trabajo presentado a las 4as Jornadas de Intercambio y Reflexión acerca de la Investigación en Bibliotecología, realizadas en La Plata del 29 al 30 de octubre de 2015. <http://www.memoria.fahce.unlp.edu.ar/ trab_eventos/ev.5287/ev.5287.pdf> [Consulta: 25 octubre 2018] 
»Odell, Jere y Ralph Gabbard. 2008. The Interdisciplinary Influence of Library and Information Science 1996-2004: A Journal-to-Journal Citation Analysis. En College \& Research Libraries. Vol. 69, no. 6, 546-564.

»Romanos de Tiratel, Susana; Graciela M. Giunti, Nora C. López, Alejandro E. Parada y María Alejandra Plaza. 2008. Revistas argentinas de humanidades y ciencias sociales: visibilidad en Bases de datos internacionales. Buenos Aires: Instituto de Investigaciones Bibliotecológicas, Facultad de Filosofía y Letras, Universidad de Buenos Aires.

»Urbizagástegui Alvarado, Rubén. 2006. Las revistas de Bibliotecología y ciencias de la información en América Latina. En Biblios: Revista electrónica de Bibliotecología, archivología y museología. No. 24. <https://dialnet.unirioja.es/ descarga/articulo/2152136.pdf> [Consulta: 6 abril 2016]

»Vallejo Sierra, Ruth Helena. 2015. Valoración y compensación social de la profesión bibliotecológica. En Códices. Vol. 11, no. 1, 37-50. <http://132.248.9.34/hevila/CodiceBogota/2015/vol11/n01/2.pdf> [Consulta: 30 octubre 2018]

"Zins, Chaim. 2007. Conceptions of information science. En Journal of the American Society for Information Science and Technology. Vol. 58, no. 3, 335-350. 